\title{
Traffic Aware Planner for Cockpit-based Trajectory Optimization
}

\author{
Sharon E. Woods ${ }^{1}$, Robert A. Vivona ${ }^{2}$ and Jeffrey Henderson ${ }^{3}$ \\ Engility Corporation, Billerica, MA, 01821 \\ and \\ David J. Wing ${ }^{4}$ and Kelly A. Burke ${ }^{5}$ \\ NASA Langley Research Center, Hampton, VA, 23681
}

\begin{abstract}
The Traffic Aware Planner (TAP) software application is a cockpit-based advisory tool designed to be hosted on an Electronic Flight Bag and to enable and test the NASA concept of Traffic Aware Strategic Aircrew Requests (TASAR). The TASAR concept provides pilots with optimized route changes (including altitude) that reduce fuel burn and/or flight time, avoid interactions with known traffic, weather and restricted airspace, and may be used by the pilots to request a route and/or altitude change from Air Traffic Control. Developed using an iterative process, TAP's latest improvements include human-machine interface design upgrades and added functionality based on the results of human-in-the-loop simulation experiments and flight trials. Architectural improvements have been implemented to prepare the system for operational-use trials with partner commercial airlines. Future iterations will enhance coordination with airline dispatch and add functionality to improve the acceptability of TAP-generated route-change requests to pilots, dispatchers, and air traffic controllers.
\end{abstract}

\section{Introduction}

$\mathrm{N}$ ASA's Traffic Aware Strategic Aircrew Request (TASAR) concept empowers aircraft operators to take a more proactive role in managing aircraft trajectories en-route. By providing pilots conflict-free route and altitude optimizations that save fuel and/or flight time, TASAR enables them to make money-saving, route-change requests that are more likely to be approved by Air Traffic Control (ATC) ${ }^{1}$ Adoption of the TASAR concept is possible in current-day operations because of the increasing availability of three technologies in the cockpit: the avionics-connected Electronic Flight Bag (EFB), Automatic Dependent Surveillance Broadcast (ADS-B) IN, and broadband internet connectivity. By advancing the aircraft's roll from passive recipient of ATC instructions to active trajectory planner, TASAR is an early initiative promoting greater operational autonomy in the National Airspace System (NAS), ${ }^{2,3}$ a strategic goal of NASA's Aeronautics Research Mission Directorate. ${ }^{4}$

In order to test the TASAR concept, NASA Langley Research Center has developed a prototype cockpit-based, flight optimization tool, the Traffic Aware Planner (TAP). ${ }^{5,6}$ Designed to operate on a variety of EFB platforms, the tool processes up-to-date data from avionics and the internet, including the aircraft's current route and state, ADS-B IN, winds, convective weather, and special use airspace schedules, to generate conflict-free, flight-optimizing, routechange advisories. The internal architecture and algorithms of TAP's main processor were derived from the NASA Autonomous Operations Planner (AOP), a flight-deck automation system developed to support aircraft self-separation research. Extensively tested and refined for over a decade, AOP generates timely, conflict-free, route-change solutions enabling an aircraft to manage its trajectory autonomously in complex, high-density en-route airspace. ${ }^{7}$ Additional components of TAP were specifically developed under the TASAR research initiative, incorporating design improvements derived from in two human-in-the-loop (HITL) simulation experiments and two flight trials.

${ }^{1}$ Principal Software Engineer, 900 Technology Park Drive, Suite 201, AIAA Member

${ }^{2}$ Chief Research Engineer, 900 Technology Park Drive, Suite 201, AIAA Associate Fellow

${ }^{3}$ Senior Research Engineer, 900 Technology Park Drive, Suite 201, AIAA Member

${ }^{4}$ Principal ATM Research Engineer, NASA LaRC, Mail Stop 152, AIAA Member

${ }^{5}$ Human Factors Research Scientist, NASA LaRC, Mail Stop 152, AIAA Member

1

American Institute of Aeronautics and Astronautics 
NASA's goals of TAP development are to increase technology readiness level (TRL) of TASAR through flight testing and operational flight trials and to promote technology insertion into flight operations. Thus, TAP has been developed to be readily adopted by a wide variety of airspace users for operational benefit, either by licensing TAP directly from NASA or working through commercial organizations that could develop TAP into a commercial product. ${ }^{8}$ NASA is currently adapting TAP to two partner airlines aircraft configurations for operational flight trials in 2017-2018 to assess its benefits for commercial operations as well as reduce any residual operational issues not identified in NASA's HITL simulation experiments ${ }^{9}$ and initial flight trials. ${ }^{10,11}$

This paper provides an overview of the functionality and capabilities of the TAP software application. It reviews the software development involved in adopting the AOP code base to the TASAR concept, meeting the system requirements and data availability conditions of TAP's multiple operational environments, and the development of a Human Machine Interface (HMI) that is both effective and intuitive to use. Future enhancements are discussed, including expanding TAP to support dispatch coordination and to improve acceptability of requests to pilots and ATC.

\section{TASAR Concept}

The TASAR concept ${ }^{1,12}$ offers onboard automation for the purpose of advising the pilot of traffic compatible route and altitude changes that would be beneficial to the flight and which are more likely to be approved by ATC. Because the concept is implemented on an individual "per aircraft" basis and uses a commercial-off-the-shelf EFB as an operating platform, it may be implemented near-term at relatively low cost, allowing for wider adoption by airspace users for immediate operational benefit. This potential for early adoption could provide the foundation for more advanced applications to emerge, leading toward greater operational autonomy in the future. ${ }^{2,3}$

Since 2012, NASA has been developing and testing the TASAR concept with the goal of providing an application that could be used by operators in the current-day NAS to optimize their flights. Early activities have included a formal definition of the concept ${ }^{12}$, an assessment of Federal Aviation Administration (FAA) certification and operational approval requirements, ${ }^{13,14}$ and a preliminary benefits analysis. The results of the benefits analysis inidcated that aircraft equipped with TASAR capabilities could reduce flight travel time from one to four minutes and fuel burn by 50 to 550 pounds. ${ }^{15}$

To verify and mature the TASAR concept, as well as facilitate its use by operators in the near-term, the TASAR project developed a software automation prototype system, the Traffic Aware Planner (TAP) software application. ${ }^{5,6}$ TAP software development has followed an iterative process, expanding and refining TAP's design and capabilities over a series of simulation experiments and flight trials, ${ }^{8}$ including:

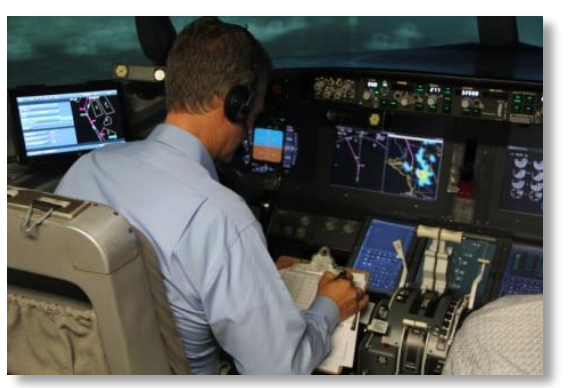

Figure 1. OPL's fixed-base flight deck simulator with the TAP software application to the pilot's left during HITL-2. Photo by M. Cover.

1. Two HITL simulation experiments, conducted in August 2013 (HITL-1) and October 2014 (HITL-2), at the University of Iowa's Operator Performance Lab (OPL) using their fixed-base flight deck simulator (see Fig. 1). Commercial airline pilots participated as evaluation pilots in both experiments. ${ }^{9}$ These experiments were designed to assess the effects of incorportating the TASAR concept, implemented via the TAP software application, into a commercial flight deck. The primary objectives of HITL-1 were to assess the effects of TASAR on pilot workload and distraction and to assess pilot usability of the HMI. During HITL-2 the primary objectives were to evaluate the HMI design update and assess the efficacy and validity of the TAP computer-based training modules.

2. Two flight trials, conducted in November 2013 (FT-1) and June 2015 (FT-2) in Advanced Aerospace Solutions's (AdvAero) Piaggio Avanti P180 flight-test aircraft, commercial airline pilots as well as the Avanti crew participated as evaluation pilots. ${ }^{10}$ Error! Reference source not found. During FT-1, TAP was installed on the UTC Aerospace Systems (UTAS) G500 SmartDisplay ${ }^{\mathrm{TM}}$ and connected to avionics via the UTAS Aircraft Interface Device (AID). The objectives of FT- 1 included the verification of live data interfaces and the validation of TAP functionality and usability in a live avionics environment. During FT-2, TAP Engine was installed on the flight test aircraft and connected to the avionics via the AID. The HMI was displayed on an $\mathrm{PPad} \mathrm{Air}^{\circledR}$. The objectives of FT-2 included an assessment of external data sources, time/fuel outcome validation methodology, pilot and controller acceptability criteria for user requests, and crew resource management. 
Development of TAP is currently focused on preparing the software application for operational flight trials with two partner airlines, Alaska Airlines and Virgin America, planned to be conducted in 2017-2018 . Data from these trials will be analyzed by NASA and the airlines to assess TASAR benefits. Current estimates of annualized benefits for each of the airlines in a fleet-wide implementation of TAP, based on simulation analysis, are savings in excess of $\$ 5$ million due to fuel, maintenance and depreciation costs. ${ }^{16,17}$ The TASAR project plans to continue expanding and maturing TAP capabilities, based on feedback and analysis of the results of FT-2 and the airline operational trials, with the goal of making it available for licensing and commercial development.

\section{TAP Operational Environment}

The following section details TAP's operating platform, data and configuration requirements, and conditions for operation.

\section{A. Platform}

To minimize installation and certification costs, TAP was designed to operate on low-cost, avionics-connect EFBs previously defined as Class $2 .{ }^{6}$ (The FAA is discontinuing designations of EFBs by "class" in favor of hardware designations of "installed" and "portable.") Since this class of EFB provides "read-only" access to onboard avionics data, it allows for TAP to be installed without requiring any costly recertification of the existing avionics. Such EFBs receive data input from cockpit avionics via a data concentrator unit such as the UTAS AID. The AID reads data from the cockpit avionics bus and sends it to the EFB using the ARINC 834-1 Simple Text Avionics Protocol (STAP). This read-only access to avionics data allows TAP to be classified as a system that has minor or no effect to safety-critical operations, further streamlining the process of FAA approval. ${ }^{13,14}$

The TAP system has been developed to operate on a variety of EFB platforms and test configurations (See Fig. 2) using a single code base:

1. UTAS G500 SmartDisplay ${ }^{\mathrm{TM}}$ EFB (Windows XP) using a simulated AID STAP data feed

2. Dell Venue ${ }^{\mathrm{TM}} 11$ Pro tablet (Windows 8) using a simulated AID STAP data feed

3. iPad Air $^{\circledR}$ tablet (iOS) connected to the UTAS Tablet Interface Module (TIM ${ }^{\mathrm{TM}}$ ) and AID (Linux)

4. Astronautics Corporation of America NEXIS ${ }^{\mathrm{TM}}$ Flight-Intelligence System EFB (Linux).

Because the current implementation of TAP assumes read-only connectivity to avionics, pilots will enter TAPgenerated route changes into their Flight Management System (FMS) manually. Future enhancements of the TAP system may allow auto-loading of the route changes via Data Communications, Aircraft Communications Addressing and Reporting System (ACARS), or the read/write capability of installed avionics.
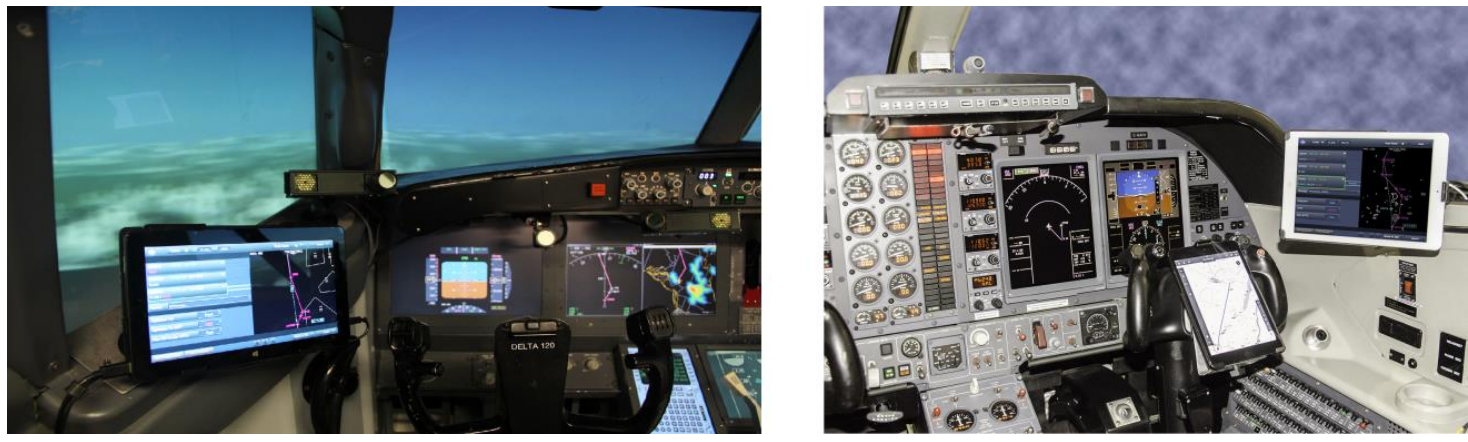

Figure 2. TAP operating on two EFB hardware configurations. Photos by M. Cover (left) and M. Haynes (right).

\section{B. Input Data}

TAP receives internal avionics data via the STAP feed and external data via an External Data Server (EDS) connected to the internet. Additional input data include an aircraft performance model and a navigational data base.

\section{Avionics Data}

TAP is designed to have access to at least the following current avionics data: time, aircraft state, Flight Control Computer (FCC) guidance mode setting, and FMS route. To provide "traffic-aware" route optimization solutions, TAP also requires traffic-aircraft state data from ADS-B IN, though traffic data is not strictly required for TAP to function. Specific data types available to TAP via the STAP feed vary among aircraft, and not all desired data types will be available in all environments. For each aircraft installation, a unique configuration specification is defined to

3

American Institute of Aeronautics and Astronautics 
identify the avionics equipment that will be providing specific data required by TAP (e.g., current longitude and latitude) and to which AID ports TAP should subscribe to receive those data.

\section{External Data Sources}

With the increased availability of broadband internet access on commercial airlines, the TASAR concept leverages data sources other than the STAP-feed from cockpit avionics. These external sources, accessible via TAP's External Data Server (EDS), enhance the validity of TAP's optimization options using data about the aircraft's operating environment. In preparation for the operational trials with the partner airlines, EDS is being configured to connect to commercial weather providers Weather Services International (Alaska Airlines) and Schneider Electric (Virgin America). Currently, TAP uses EDS in flight to gather up-to-date data for three-dimensional (3D) gridded winds, convective weather, and Special Use Airspace (SUA) schedules. Several other external data types have been proposed for future integration, including turbulence, icing, and volcanic ash data. EDS has also been proposed as a possible agent for relaying communications to and from dispatches to enhance coordination of TASAR-based route changes.

\section{Aircraft Performance Model}

To generate accurate trajectory predictions, TAP uses aircraft performance tables specific to the hosting aircraft type. The source of these data varies among manufacturers and in some cases may be proprietary. For the operational trials, TAP has been configured to interpret performance data in the formats provided by the partner airlines. The absence of a pervasive industry standard format for cruise-phase performance data means that adaptation of either the TAP software or the performance dataset will be required for each installation.

\section{Navigation Database}

To facilitate today's voice communications between pilots and controllers, TAP constrains its route-change solutions to using named waypoints. Thus, TAP installation includes a navigation database of waypoints based on the ARINC 424 standard corresponding to the same database cycle used by the aircraft's FMS. Coorindated updates to the databases will assure that waypoints suggested by TAP will be recognized by the FMS system and by ATC. It will also assure that TAP will be able to recognize all the waypoints of the active route currently used by the FMS for guidance. TAP also uses the navigation database to obtain boundaries of SUA and the destination airport's altitude (required for trajectory prediction but not available from the STAP feed).

\section{Conditions for Operation}

TAP is intended for use during the en-route, cruise portion of the flight, when pilot workloads are lightest and opportunities for route optimization are most prevalent. However, it is capable of generating optimizations throughout the upper climb, cruise, and early descent phases of flight. To avoid interfering with terminal operations, TAP is configured to be operational only when the ownship aircraft is above 10,000 feet and a configurable distance from destination. Since opportunities for optimization diminish close to the destination, TAP usage is expected to be discontinued late in the flight. ${ }^{15}$

\section{TAP System Description}

\section{A. TAP System Architecture}

The TAP system consists of four executable components: TAP Engine (the main processor), TAP Display (the human machine interface), Display Adaptor (for internal communications), and EDS. Following a discussion of basic system architecture, a description of each is provided in the upcoming subsections.

All four components are designed to operate on Linux and Windows operating systems. TAP Display also operates on iOS and (potentially) Android operating systems. This separation of executables and cross-platform capabilities allow TAP to be installed on a diversity of EFB hardware systems and configurations. For instance, in the UTAS Tablet EFB system (i.e., the Alaska Airlines configuration), TAP Display will operate on an iPad Air ${ }^{\circledR}$, with the processing units operating on the UTAS Linux-OS TIM ${ }^{\mathrm{TM}}$ and AID. For the Astronautics NEXIS ${ }^{\mathrm{TM}}$ EFB (Virgin America configuration), all the executables will reside on the single Linux-OS NEXIS ${ }^{\mathrm{TM}}$ unit.

Figure 3 depicts the TAP system architecture and how it accesses data in different configurations. The EFB will have a STAP-formatted data input feed from either a data concentrator emulator (in simulation environments) or the aircraft AID, which provides the TAP Engine the required avionics data. EDS, which provides formatted data to the TAP Engine from sources other than the avionics, requires access to the internet via an Ethernet connection to the aircraft's broadband internet provider system. Other possible data sources, such as satellite weather systems, could also be accessed by the EDS using the EFB's Ethernet connection as long as they reside on the same local area network

4

American Institute of Aeronautics and Astronautics 
as EDS. EDS can provide data to multiple instances of TAP (e.g. captain and first officer). The TAP Engine communicates with TAP Display via the Display Adaptor, which adapts the TAP system to the communication

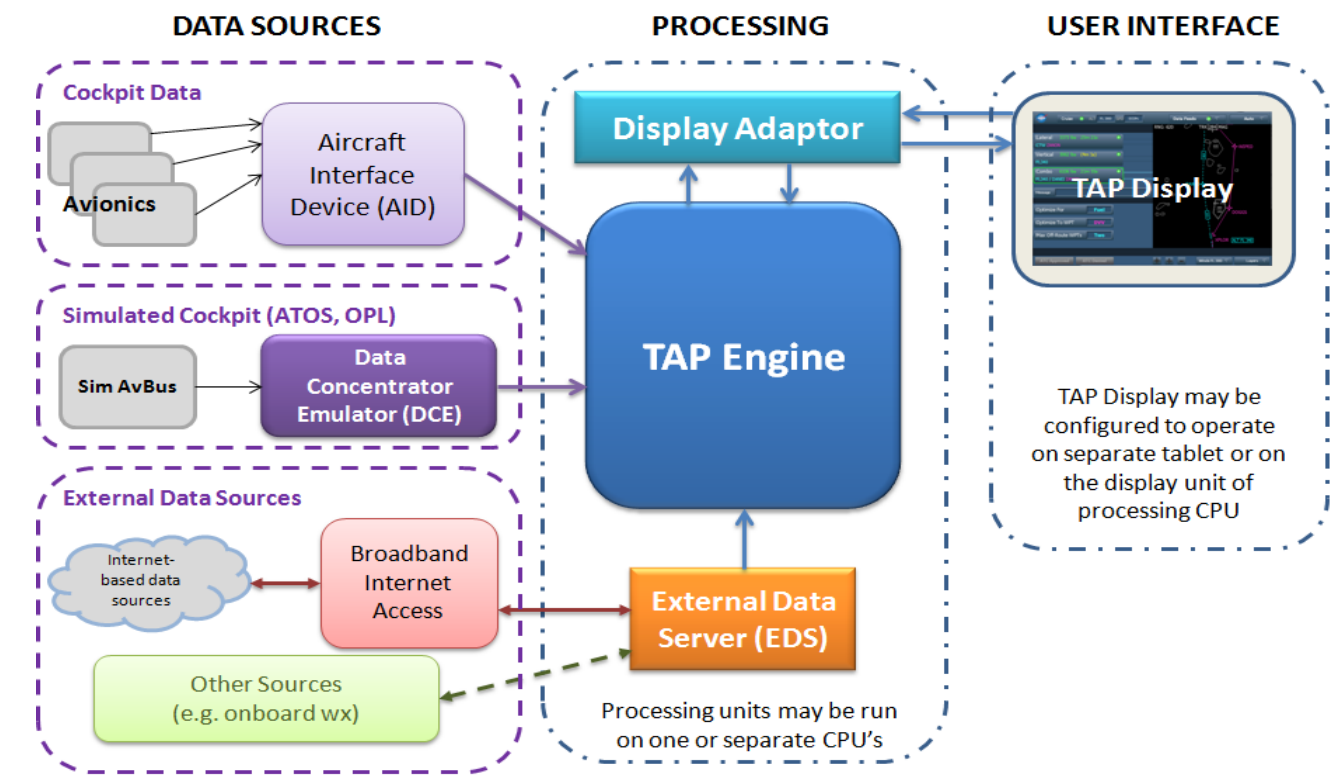

Figure 3. TAP System Architecture.

protocol available on the EFB hardware. Detailed descriptions of each component follow.

\section{TAP Engine}

TAP Engine is the main processor of TAP. It accepts and reads all data inputs, reformats them as needed for internal use, performs all processing necessary to generate route optimization advisories, and responds to all pilot commands on TAP Display that affect processing. Its central architecture, algorithms, and performance are derived from NASA's Autonomous Operations Planner (AOP), an airborne trajectory management decision-support tool developed for research into advanced concepts of airborne operational autonomy and self-separation. ${ }^{7}$ Whereas AOP's primary function is to detect ${ }^{18}$ and resolve ${ }^{19}$ conflicts in a fuel-efficient manner, TAP Engine's main functionality is to continuously probe for candidate route changes (including altitude changes) that optimize fuel burn, flight time, or trip cost (combining trip fuel and time according to a given Cost Index) across the entire route while avoiding the creation of conflicts with traffic, weather, and SUA. Though TAP is an optimization tool and not a self-separation tool, it is embedded with the deep heritage of AOP's extensively tested self-separation and four-dimensional, airbornetrajectory-management capabilities.

The development of TAP Engine involved several augmentations to AOP's functionality, including de-coupling it from the mode controls of NASA's simulation environment to operate as a stand-alone system, adding the capability to read avionics data from the STAP-feed, and adding maneuver templates to the pattern-based genetic algorithm ${ }^{19,20}$ to create the optimized route changes. ${ }^{5} \mathrm{TAP}$ also includes logic to limit route-change solutions to named waypoints such that they can be verbally requested from ATC, ${ }^{21}$ whereas AOP was free to use arbitrary latitude/longitude positions for waypoints.

The trajectory generator (TG) function used in AOP was designed for the high-fidelity requirements of selfseparation and integration with an aircraft's FMS. In order to make the function more adaptable for TAP's operational use on a wide variety of aircraft types and to tailor it to the appropriate fidelity for TAP's route optimization function, a new TG was developed specifically for TAP. With a system architecture based on the abstraction technique of separating mathematical modeling from behavioral modeling,Error! Reference source not found. the TAP TG has the ability to be expanded to handle different aircraft modeling data and behaviors with one code base. An initial version of the TAP TG was tested during FT-2, modeling the behavior of the Piaggio Avanti turboprop aircraft using performance data extracted from the manufacturer's pilot flight planning handbook. In preparation for the operational trials with Alaska Airlines and Virgin America, the TAP TG is being expanded to model the Boeing 737-900ER and Airbus A319 and A320 using performance data provided by the airlines and aircraft manufacturers.

5

American Institute of Aeronautics and Astronautics 


\section{Display Adapter}

The Display Adapter handles communications between TAP Engine and TAP Display, using the protocols specific to the EFB hardware configuration hosting the TAP software. It receives all display data communication from TAP Engine, adapts it to the communication protocol of the hardware configuration, and sends it to TAP Display. Likewise, it handles all pilot commands from TAP Display and relays them to TAP Engine. In configurations where TAP Display is operating on a tablet device, the Display Adaptor also re-synchronizes connections with TAP Display after it has been put to sleep or pushed into background operation while another application is in use.

\section{TAP Display}

TAP Display is the HMI which enables the interaction between the pilot and the software. It displays TAP's optimization solutions, time/fuel outcomes, conflict characteristics, as well as additional information about the state of the system. The HMI also accepts and implements entry of all pilot control instructions and is adaptable to many EFB configurations, operating systems, display aspect ratios, and orientations (see Fig. 4). The HMI was developed through an iterative human-
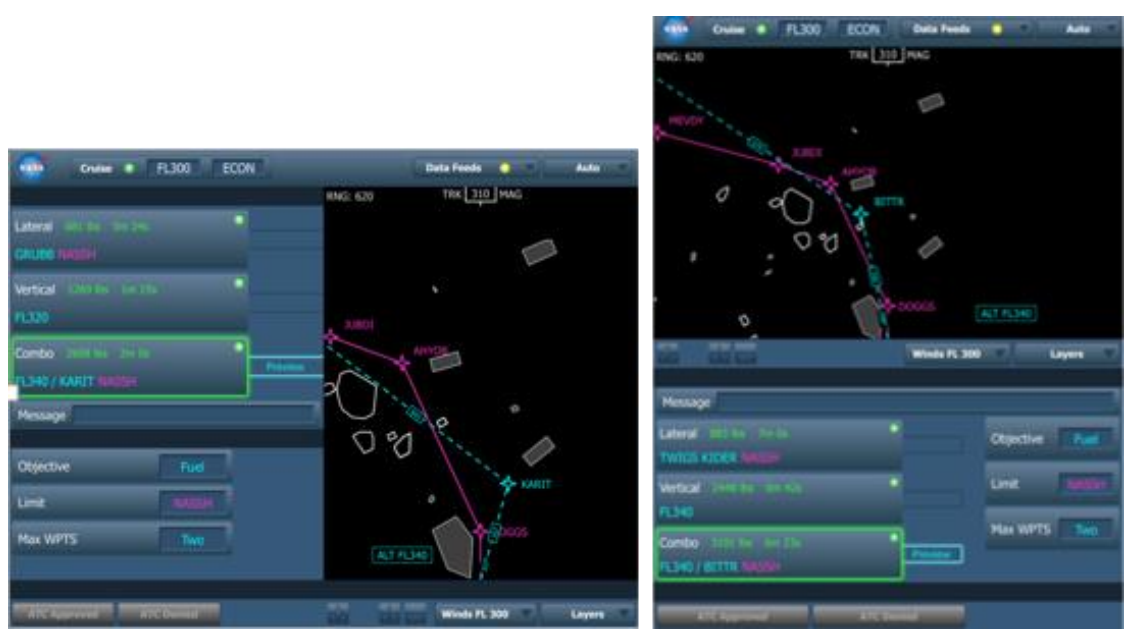

Figure 3. Depiction of TAP Auto Mode in the 4/5 aspect ratio used by Apple iPad ${ }^{\circledR}$ tablets in both landscape and portrait orientations.

factors design process and subsequesntly enhanced over the course of the TASAR project. Incorportatiing the analysis results of objective and subjective data collected from evaluation pilots during HITL-1 and FT-1, human factors principles were applied throughout the development of all the HMI features to enhance usability and situation awareness, without significantly affecting pilot workload. Recent improvements include consolidating the total number of screens, adding visual feedback to indicate the best overall optimization solution, incorporating tabletspecific styling guidelines including menu-scrolling capabilities, and providing a more user-friendly and intuitive interface for manually entering route changes. The updated HMI design was tested and evaluated by pilots during HITL-2 and FT-2 and assessed for its effects on pilot perceived workload and situation awareness, and pilot usability and acceptability of the interface. Pilot ratings of comprehensibility, usefulness, and usability of the updated HMI were very high, pilot perceived workload was low, and situation awareness of normal cockpit operations was not effected. ${ }^{9}{ }^{10}$,Error! Reference source not found.

A key element of the HMI is the Visualization Panel (Vis Panel), a moving-map display providing graphic visualization of the proposed route changes and contextual information used or computed by TAP. The Vis Panel allows a pilot to quickly assess the geometric nature of the displayed TAP solution and to answer immediate questions such as whether the initial turn is right or left and how far the waypoints of the route change are relative to one another. In cases where the route change has a conflict, it indicates which portion of the route change has the conflict and, if applicable, the geometry of the hazard that is causing the conflict (see Fig. 5). Selectable layers of area hazards (e.g., weather, SUA) and wind data (see Fig. 6) may be displayed at the pilot's discretion using menu
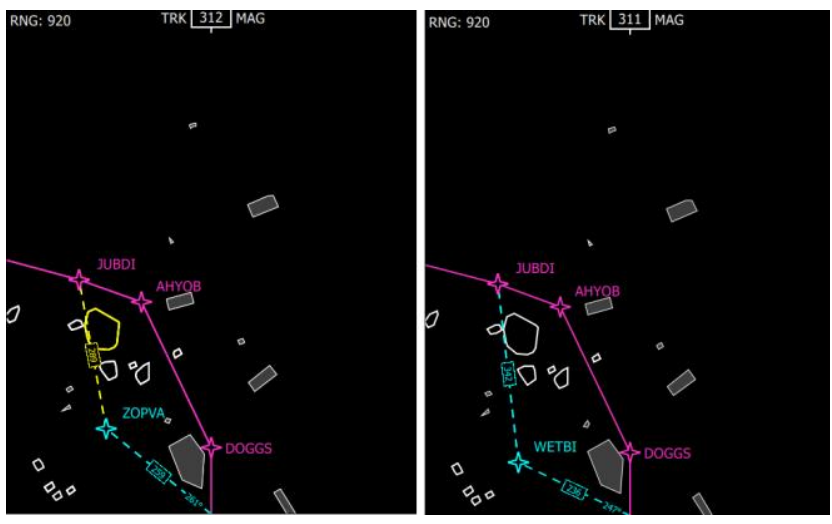

Figure 5. TAP Vis Panel depicting two potential route changes in Manual Mode, one with a conflict, one without. controls. 


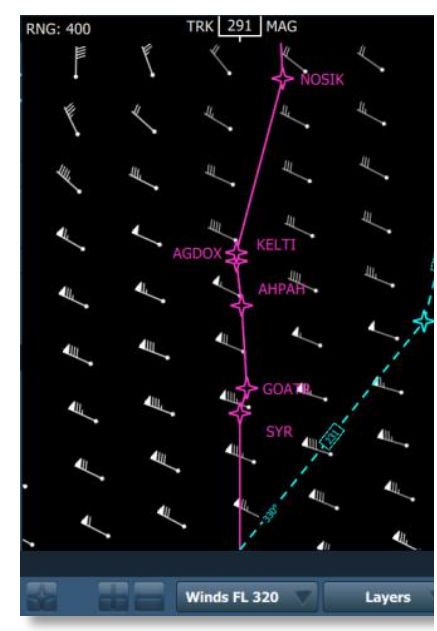

Figure 4. TAP Vis Panel.
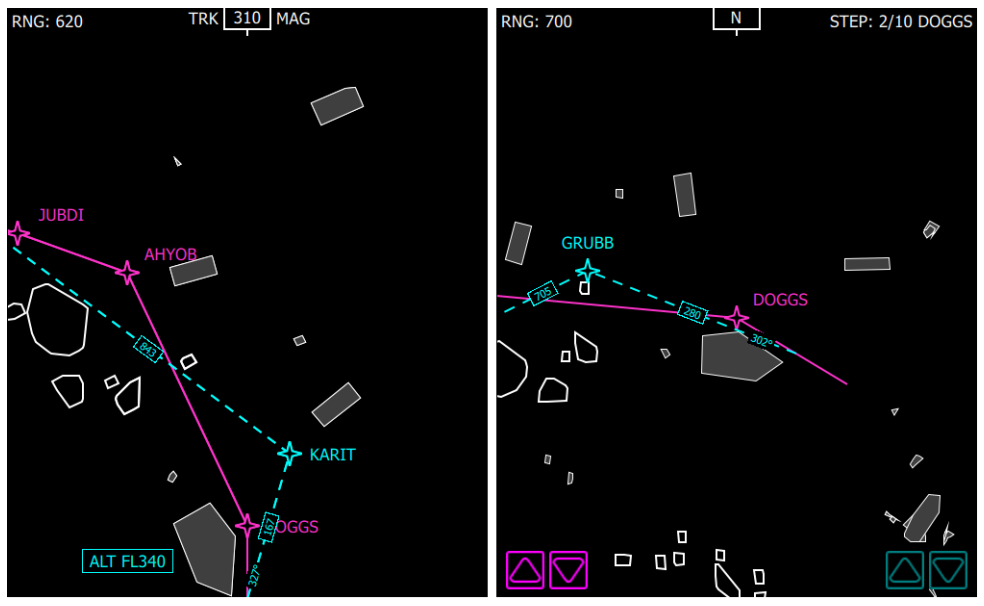

Figure 5. TAP Vis Panel depicting Normal (track-up) View (left) versus Step View (right).

The Vis Panel has two viewing projections: a track-up projection similar to navigation displays, and a north-up "step" view that allows the pilot to step waypoint-by-waypoint through the current route or the route change. In both projections, the graphical display does not show current ownship position due to certification reasons (see Fig. 7). The Vis Panel is used in conjuction with interactive tools for manually building route changes, allowing the pilot to choose off-route waypoints by touching a desired location directly on the map and allowing TAP to find the nearest named waypoint to the location touched.

\section{External Data Server (EDS)}

The External Data Server is a separate process that handles the connection, download, and processing of data from sources other than the ownship's avionics via the STAP feed. During FT-2, EDS downloaded three types of data: winds and temperature aloft data from the Rapid Refresh (RAP) service available via internet from the National Oceanic and Atmospheric Administration; SUA status and schedule via the internet from the FAA SUA website; and convective weather polygons from a NASA-based server connected to commercial weather data provider, WSI.

EDS is designed to be expandable to incorporate numerous other data sources. Adaptation to new data sources requires analysis of the source data and what subset of the available data is relevant to a single instance of TAP, given the aircraft's location and active route. EDS connects to the data source, requests the data (or appropriate subset), converts it into TAP format, and passes it to TAP Engine.

Currently, EDS is an on-board application only. Future enhancements of EDS include a proposal to introduce a ground-based server component to handle the larger task of data management of internet sources, from which the onboard version of EDS will request a data subset appropriate for its single flight.

\section{B. TAP Functionality and Capabilities}

TAP has two modes of operation: Auto Mode and Manual Mode. In Auto Mode, TAP continually monitors for optimization opportunities and generates conflict-free route-change solutions that meet the aircrew's optimization objective (e.g., minimize fuel burn). In Manual Mode, TAP provides functionality to assist aircrews in manually creating route changes that meet aircrew objectives while increasing the likelihood of ATC approval.

\section{Auto Mode}

When in Auto Mode, TAP continuously generates a set of optimization solutions at a nominal one-minute update rate, giving the pilot a reasonable amount of time to determine whether they will pursue requesting the proposed route change from ATC. TAP generates three types of optimization solutions:

- Lateral: A path-change maneuver, with up to two off-route waypoints added before reconnecting to the active route at a waypoint. All waypoints are named, allowing for easier verbal communication with ATC.

- Vertical: A cruise-altitude change, with no change to the existing lateral path.

- Combo: A combined maneuver that includes both a cruise altitude change and a path change. This is a unique and independent solution, not just a composite of the lateral-only and vertical-only solutions.

Pilots have the option to configure TAP Auto Mode in its generation of optimization solutions. These configuration settings include:

7

American Institute of Aeronautics and Astronautics 
- $\quad$ Limiting the maximum number of off-route waypoints $(0,1$, or 2$)$

- Specification of optimization objective (e.g., minimize fuel burn)

- Defining the optimization limit, a waypoint on the active route beyond which TAP should not modify the route.

Figure 8 depicts TAP's HMI in Auto Mode. Because Auto Mode is intended to be advisory information only and to run continuously without distracting the pilot, the HMI was designed to require no interaction from the pilot unless they want to change settings or select an optimization solution for further consideration.

TAP Auto Mode will search for route optimization solutions (including altitude) once a minute. For each optimization solution, TAP calculates the predicted outcomes of the route change, i.e., the effect on fuel and flight time. These outcomes are refreshed every ten seconds.

For each set of route optimization solutions, TAP calculates which one is the "best" based on the pilot's current optimization objective setting. The HMI indicates this best solution by displaying a green border around the appropriate solution button and automatically "Previews" that solution on the Vis Panel so that the pilot can quickly assess the nature, direction, and distances associated with the proposed maneuver. If the pilot desires to preview one of the other solutions on the Vis Panel, they may touch the desired solution button to shift the Vis Panel preview to the selected solution.

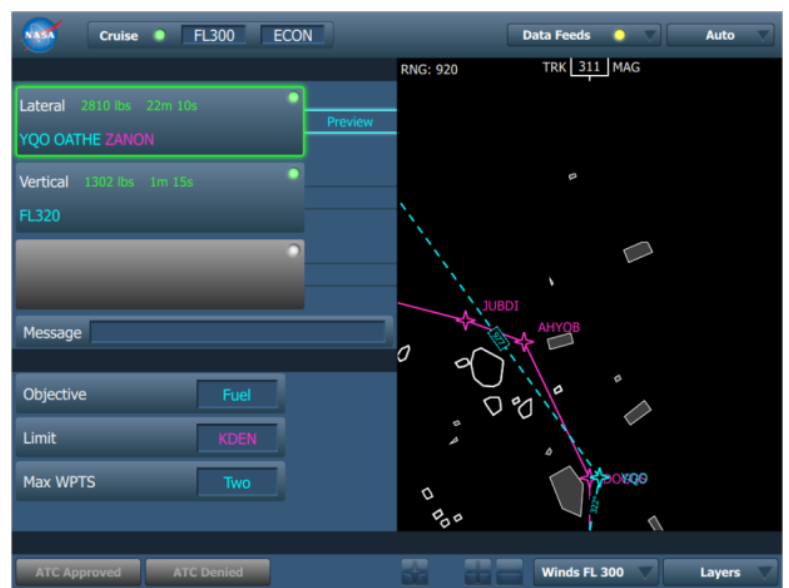

Figure 7. TAP Auto Mode in a cycle where no conflict-free Combo solution was found that met optimization goals.

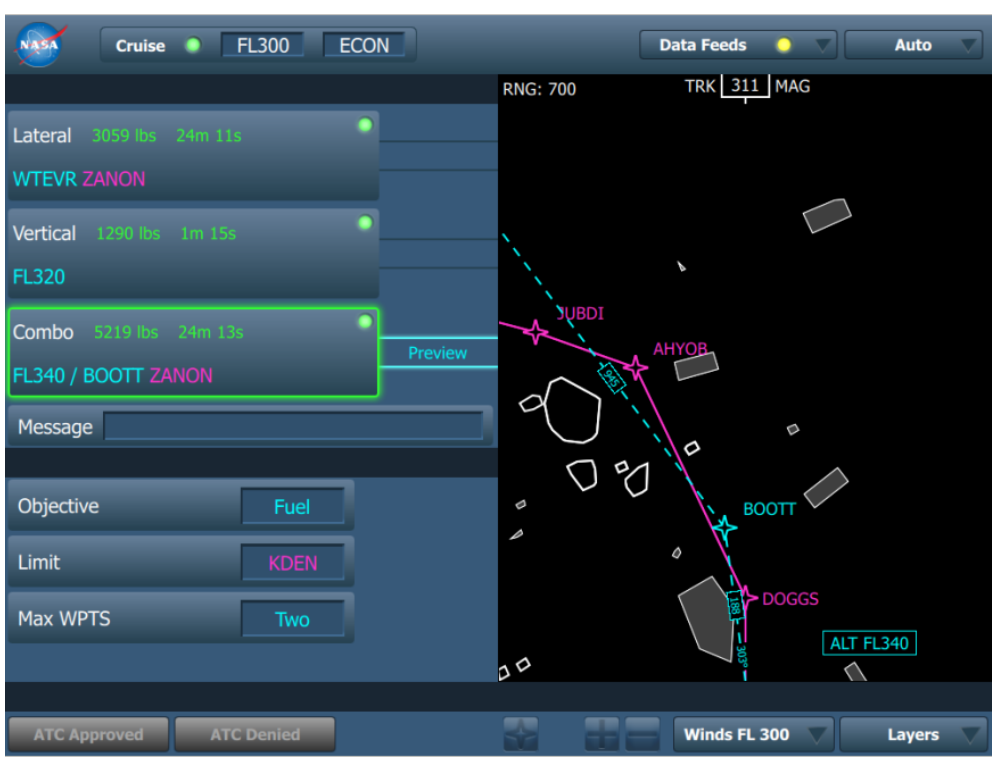

Figure 6. TAP Auto Mode screen.

solution for that type will be grayed out for that generation cycle (see Fig. 9). For each maneuver type, the algorithm will not return a solution that conflicts with traffic or airspace hazards at the time it was computed or that does not achieve a savings in the optimization objective. All three solutions grayed out indicates that the aircraft is already on the most optimal route currently available.

Lateral and Combo solution types may include up to two off-route waypoints (i.e., waypoints not already on the active route). If the pilot wishes only to see solutions that are "directs" (i.e., proceeds directly to a downstream waypoint on the active route) or have just one off-route waypoint, they may limit the number of off-route waypoints using the "Max WPTS" selection. TAP seeks to minimize the number of off-route waypoints used, not to exceed the 
maximum setting. For example, if Max WPTS is set to two off-route waypoints, but a direct-to-waypoint is the best conflict-free lateral solution, that is the solution that TAP will produce.

If the aircrew sees an optimization solution they feel may be a candidate for an aircrew request to ATC, they can "freeze" the optimization by selecting it (see Fig. 10). Freezing a solution prevents TAP from overwriting the solution in the next automatic generation cycle. Once selected, the solution will remain frozen until the pilot cancels the selection by selecting "Release," upon which the latest set of optimization solutions will then be displayed.

Though TAP produces solutions that are originally conflict free, conflicts can develop during the time the solution is frozen. TAP therefore re-evaluates a selected (i.e., frozen) optimization solution every ten seconds, checking if the

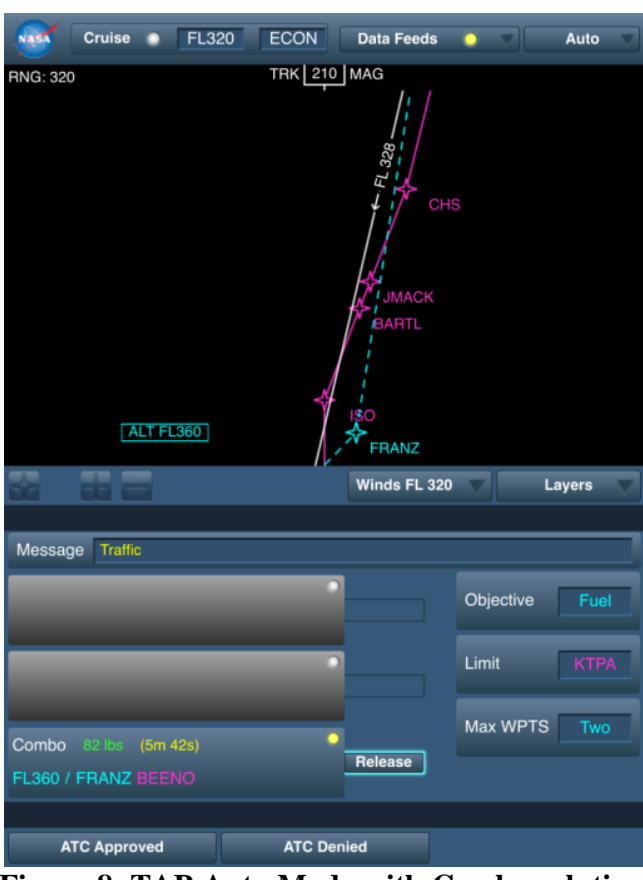

Figure 8. TAP Auto Mode with Combo solution selected, and evaluated to have developed a traffic conflict while frozen. route change is still conflict-free and if it is still considered reasonably navigable (i.e., lies within preset geometric ranges as described in Ref. 21). If the re-evaluation determines that the route change would conflict with convective weather, SUA, and/or traffic, it turns the indicator light of the selected optimization to yellow, and provides a message describing the conflict(s) in the Message window.

The conflict is also displayed on the Vis Panel. For example, in Fig. 10, TAP detected a traffic conflict for the selected optimization. Indications of traffic conflict on the Vis Panel show only the track direction and flight level of the traffic, not its location.

Each re-evaluation of the selected optimization solution will also update the outcomes of the route change. This allows the pilots to monitor if the route change remains beneficial while they consider whether to make an aircrew request to ATC. These outcomes will be generated as long as the route change is still considered reasonably navigable.

Once a pilot is satisfied that a selected optimization solution is acceptable (procedures may dictate verifying operational acceptability using certified systems such as the FMS and onboard weather radar), they may proceed with communicating the route change request to ATC. When ATC response is received, the pilot selects ATC ACCEPT or ATC DENIED to record the outcome for later analysis, if desired.

\section{Manual Mode}

TAP Manual Mode enables the pilot to directly enter a desired route change into TAP and have it evaluated for fuel and time outcomes and any predicted conflicts with known traffic or area hazards. This mode allows the pilot to puruse objectives other than the Auto Mode Objective settings, such as avoiding reported turbulence. If TAP detects a conflict in a manually entered route change, it allows the pilot to alter the route change so that TAP can re-evaluate it. Manual Mode accepts route changes of the same three types generated in Auto Mode: Lateral, Vertical, and Combo.

Manual Mode's functionality has two objectives: provide a flexible, user-friendly mechanism for the pilot to manually enter and edit a route change, and evaluate the route change for conflicts and fuel and time outcomes. Fig. 11 shows an example of a lateral-only maneuver being created in Manual Mode. The upper left section of the screen provides the controls for entering a route

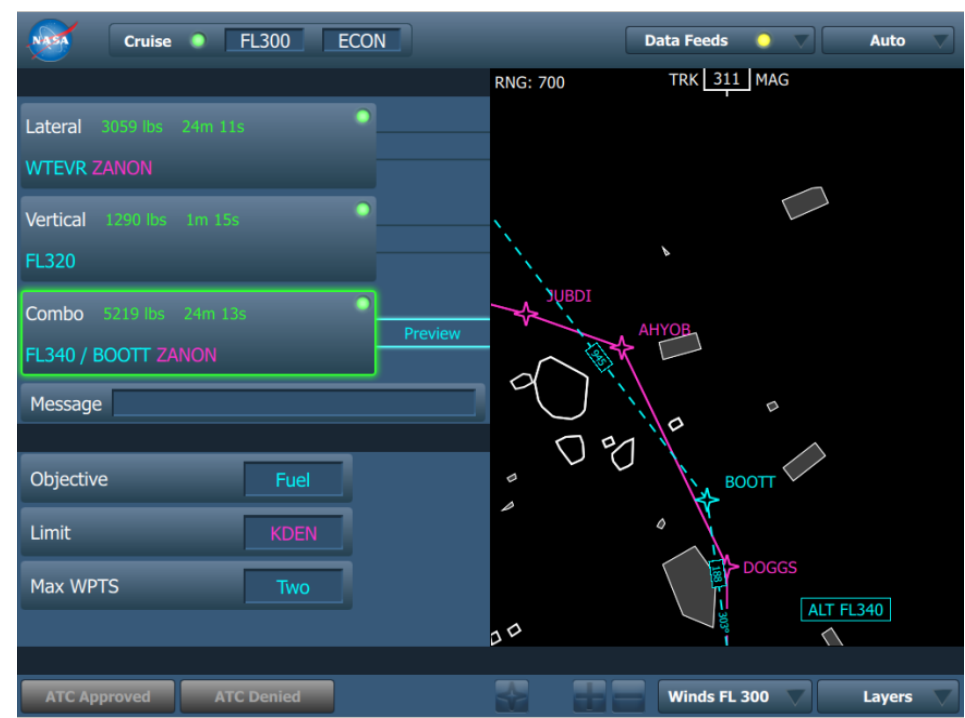

Figure 9. TAP Manual Mode screen.

\section{9}

American Institute of Aeronautics and Astronautics 
change. The lower left section shows the route change that is currently being evaluated, its time and fuel outcomes, and any conflicts detected for the entered route change. The Vis Panel also displays the route change and conflict information, if applicable.

TAP refreshes the evaluation of the manual route change every ten seconds or if there is any change to its specifications. The refresh updates the outcomes and rechecks if the route change is still conflict-free and considered reasonably navigable. The pilot may leave a manual evaluation displayed as long as they wish, but if TAP determines it is no longer reasonably navigable, no outcomes are calculated.

To support flexible route change entry, TAP provides several controls to expedite the process for the pilot. As stated earlier, the pilot may define lateral-only, vertical-only, or combo route changes. TAP will evaluate a manual optimization as soon as the following criteria are met:

- Lateral maneuvers (if any) include specification of a reconnect waypoint on the active route

- Vertical maneuvers (if any) include a new flight level other than the current cruise altitude

To ensure that the pilot only designates a reconnect waypoint that is on the active route, the HMI displays a dropdown list of the active route's waypoints, but the pilot is also permitted to select the reconnect waypoint directly on the Vis Panel. If this reconnect waypoint is subsequently removed from the FMS active route while the TAP Manual Mode screen is active, TAP will indicate that it is no longer able to evaluate. For vertical maneuvers, the HMI displays a scrollable list of flight levels from which the pilot may choose the desired flight level.

When designating off-route waypoints, the pilot may not necessarily know the names of the waypoints in the vicinity of the route change. TAP allows a pilot to request the nearest waypoint to any location touched on the Vis

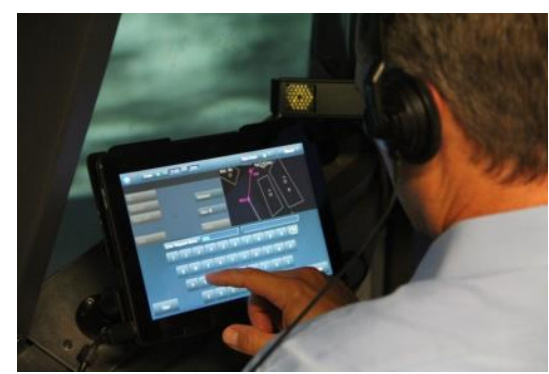

Figure 10. Pilot using Keyboard to enter a waypoint name in Manual Mode. Photo by M. Cover. Panel and to change this location repeatedly until they find a waypoint that fits their needs. If the pilot knows the actual name of the waypoint they wish to use, an on-screen keyboard allows the pilot to enter the name manually, as shown in Fig. 12. If the pilot enters a waypoint name that does not exist in the navigation database, TAP will inform the pilot of the invalid name.

When a pilot is constructing a route change with two off-route waypoints, the same tools (i.e., Vis Panel and keyboard) may be used to enter a second waypoint.

\section{Startup Checklist and Performance/Constraints}

TAP requires certain data regarding the FMS's route in order to build accurate trajectories. Route data, received through the STAP feed, that conforms to ARINC 702A-1 or ARINC 702A-3 specification standards contain much of the required data but with some key exceptions such as cruise altitude, cruise speed, waypoint names, and destination. Other route data that are not available, such as waypoint altitude and speed constraints, do not prohibit TAP from generating trajectories, but affects their accuracy.

In order to address these data gaps, TAP includes an initialization phase where the pilot enters or verifies the missing data needed for TAP to successfully operate via the Startup Checklist (see Fig. 13). Once the pilot completes this process, they will not be required to complete the checklist again. Updating the settings based on route changes during the flight can be accomplished via the Performance/Constraints (Perf/Con) screen (see Fig. 14).

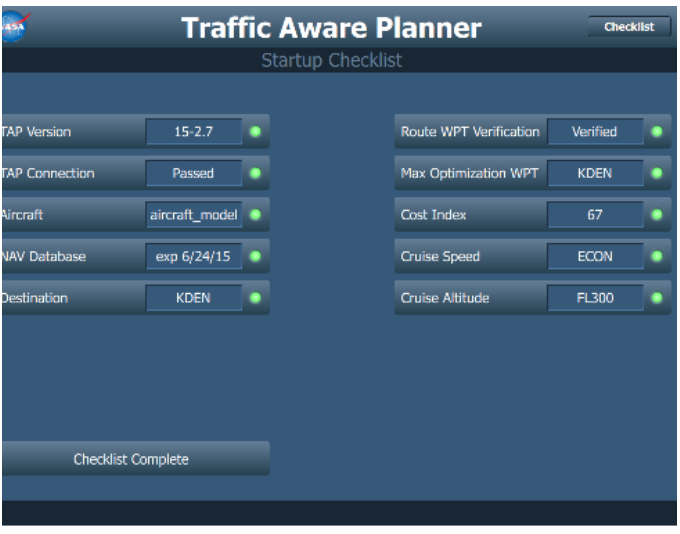

Figure 11. Startup Checklist screen.

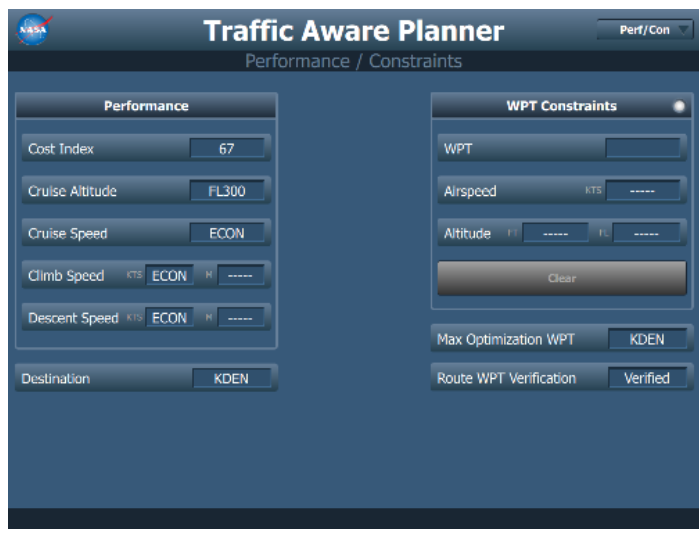

Figure 14. Performance/Constraints screen.

10

American Institute of Aeronautics and Astronautics 
Whenever possible, TAP will attempt to alleviate the work necessary for the pilot to enter these missing data values. For instance, TAP will use its waypoint search capability to guess which named waypoint in its navigational database coincides with the latitude and longitude location for the waypoint provided in the data feed. Because the location values may not correlate exactly with the database location, the pilot is asked to verify these names. If a name has not yet been verified, a symbol is displayed indicating it is TAP's "best guess" until the pilot verifies it. However, even if the waypoint is incorrectly named, it will still be used correctly for trajectory prediction.

In Auto and Manual modes during cruise, TAP monitors the aircraft's current state data and indicates to the pilot if their Perf/Con setting for cruise altitude and cruise speed match the current state (see Fig. 15). If a discrepancy is detected, the indicator will be displayed yellow, and the pilot can access menus to correct these settings directly from the Auto and Manual Mode screens. Alternatively, the changes can be made via the Perf/Con screen. This monitor is not implemented during climb and descent.

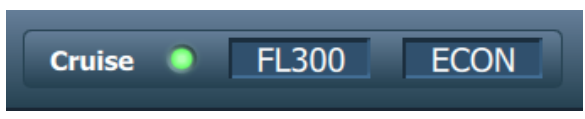

Figure 12. Monitor on Auto and Manual Mode screens indicating if latest aircraft state data matches the TAP's current Perf/Con settings for cruise altitude. and

\section{Future Developments}

Several future capabilities are in consideration to be added to TAP during or after its initial deployment for operational trials. These include capabilities to better support aircrew-dispatch coordination, process and display additional area hazards (e.g., turbulence), integrate data sources available via the FAA's System Wide Information Management (SWIM), add ATC sector awareness for evaluating acceptability of route-change requests, use data from onboard weather radar, and use Data Communications to issue change requests to ATC. Two such capabilities are briefly described.

\section{Dispatch Coordination}

Some TAP-generated route-optimization solutions may be extensive enough to require coordination with airline dispatch before aircrews make the request to ATC. A typical threshold requiring dispatcher coordination is if the proposed route change deviates laterally by more than 100 nautical miles or vertically by 4000 feet or more from the previously coordinated route. Two proposed capabilities are anticipated to enhance dispatch coordination: 1) compute and indicate to the pilot whether a TAP solution exceeds authority limits and requires coordination with dispatch, based on quantifying its differences from a baseline trajectory, and 2) enable route-change coordination messages between dispatch and aircrew via ACARS or other internet-based communications. The latter capability would allow the dispatcher to view the proposed route change and use additional information and tools they might have to assess its acceptability. Dispatchers and captains are jointly responsible for the flight, and enhancing the coordination process via digital data exchange will enable greater effectiveness and less workload in making joint decisions.

\section{ATC Sector Awareness}

During FT-2, two analysts conducted onsite observations of controllers interacting with the test aircraft and normal traffic, and interviewed controllers to assess ATC acceptability of TAP requests.Error! Reference source not found. Among the assessment's recommendations for improving TAP request acceptability were several factors relating to sector boundaries, including avoiding making requests when the aircraft is close to being handed off to the next sector and not making requests that result in unnecessary "point outs," an ATC procedure in which a controller in charge of an aircraft that flies closer than 2.5 nautical miles from a sector boundary is required to call the controller of the other sector to have them monitor the aircraft as well. Currently, pilots do not have any visual graphical clues of sector boundaries, and the interviewed controllers were open to the idea of them obtaining such awareness through the TAP's Vis Panel. Another TAP capability under consideration would be to constrain route-change solutions near sector boundaries to not generate point-out situations. However, adding ATC sector awareness to TAP would require access to sector boundary data, which is not readily available and can be dynamic as sectors are occasionally combined. Thus, sector-aware capabilities will likely be deferred until the data is available and operational trials confirm the operational benefits of these capabilities.

\section{1}

American Institute of Aeronautics and Astronautics 


\section{Conclusions}

The TAP system is an operationally deployable, prototype software application of the TASAR concept that is adaptable for use on a wide variety of EFB hardware and aircraft configurations. Starting in 2017, it will be used for operational-use trials on commercial flights of two partner airlines, Alaska Airlines and Virgin America. Results from these trials will provide data on the usability and benefits of the TASAR concept, as well as feedback on the functional capabilities of TAP when used in airline operations.

Development of the TAP system has enabled TASAR to be evaluated in two HITL simulation experiments and two flight trials. The HMI has been highly rated and verified ready for operation use by airline pilots, and TAP's ability to receive and process data from onboard and internet-based sources has been verified. As the TASAR project continues, it is expected that TAP capabilities will be expanded to include dispatch coordination, increased pilot awareness of ATC sectors, and the integration of additional data sources to improve the acceptability of its route optimization solutions to pilots, dispatchers, and ATC.

\section{Acknowledgments}

This project was funded by NASA under contract NNL12AA06C. David Roscoe, Dr. David A. Karr, Stephen DePascale, Brendan LeFebvre and Andres Danziger of Engility Corporation all contributed to the development of TAP. John Maris and Marie-Hélène Larose of Advanced Aerospace Solutions provided technical support in adapting TAP to operate on AdvAero's Piaggio P180 Avanti test aircraft. Mark Haynes of AdvAero was the test director of both flight trials and assembled the final reports of their findings. Matthew Cover of University of Iowa Operator Performance Laboratory provided technical support for adapting TAP to operate in the OPL's flight deck simulator.

\section{References}

${ }^{1}$ Ballin, M.G. and Wing, D.J., "Traffic Aware Strategic Aircrew Requests (TASAR)", AIAA-2012-5623, AIAA $12^{\text {th }}$ Aircraft Technology, Integration, and Operations Conference (ATIO), Indianapolis, IN, USA September 2012.

${ }^{2}$ Cotton, W.B., Hilb, R., Koczo, S., and Wing, D.J., "A Vision and Roadmap for Increasing User Autonomy in Flight Operations in the National Airspace", accepted to AIAA $16^{\text {th }}$ Aviation Technology, Integration, and Operations (ATIO) Conference, Washington, DC, USA, June 2016.

${ }^{3}$ Wing, D.J. and Cotton, W.B., "For Spacious Skies: Self-Separation with 'Autonomous Flight Rules' in US Domestic Airspace", AIAA-2011-6865, AIAA Aviation Technology, Integration, and Operations (ATIO) Conference, Virginia Beach, VA, September 2011.

${ }^{4}$ NASA Strategic Plan 2014, http://www.nasa.gov/sites/default/files/files/2014_NASA_Strategic_Plan.pdf

${ }^{5}$ Woods, S., Vivona, R., Roscoe, D., Lefebvre, B., Wing, D., and Ballin, M., “A Cockpit-based Application for Traffic Aware Trajectory Optimization," AIAA-2013-4967, AIAA Guidance, Navigation and Control (GNC) Conference, Boston, MA, USA, August 2013.

${ }^{6}$ Wing, D. J., Ballin M., Koczo, S., and Vivona, R. A., "Developing an On-Board Traffic-Aware Flight Optimization Capability for Near-Term Low-Cost Implementation", AIAA-2013-4231, AIAA $13^{\text {th }}$ Aviation Technology, Integration, and Operations (ATIO) Conference, Los Angeles, CA, USA, August 2013.

${ }^{7}$ Karr, D.A., Vivona, R.A., Roscoe, D.A., and Wing, D.J., "Autonomous Operations Planner: A Flexible Platform for Research in Flight-Deck Support for Airborne Self-Separation”, AIAA-2012-5417, AIAA $12^{\text {th }}$ Aircraft Technology, Integration, and Operations Conference (ATIO), Indianapolis, IN, USA, September 2012.

${ }^{8}$ Wing, D. J., "Achieving TASAR Operational Readiness", AIAA-2015-3400, AIAA $15^{\text {th }}$ Aviation Technology, Integration, and Operations (ATIO) Conference, Dallas, TX, USA, June 2015.

${ }^{9}$ Koczo, S., Burke, K., and Wing, D., "TASAR Human Factors Certification and Operational Approval Analysis and Assessments: A Summary Report", NASA Technical Memorandum submitted for publication, 2016.

${ }^{10}$ Maris, J. M., Haynes, M. A., Wing, D.J., Burke, K.A., Henderson, J., and Woods, S.E., "Traffic Aware Planner (TAP) Flight Evaluation", AIAA-2014-2166, $14^{\text {th }}$ Aircraft Technology, Integration, and Operations Conference (ATIO), Atlanta, GA, USA, June 2014.

${ }^{11}$ Burke, K., Wing, D., Haynes, M., Maris, J., Enea, G., Henderson, J., Idris, H., Vivona, B., and Woods, S., "Flight Trial Evaluations of the Traffic Aware Planner", NASA Technical Paper submitted for publication, 2016.

${ }^{12}$ Henderson, J., "Traffic Aware Strategic Aircrew Requests (TASAR) Concept of Operations", NASA/ CR-2013218001, May 2013.

${ }^{13}$ Koczo, S. "Analysis of Operational Hazards and Safety Requirements for Traffic Aware Strategic Aircrew Requests (TASAR)”, NASA/CR-2013-218002, May 2013.

${ }^{14}$ Koczo, S. and Wing, D., "An Operational Safety and Certification Assessment of a TASAR EFB Application", accepted for publication, 32 ${ }^{\text {nd }}$ Digital Avionics Systems Conference, Syracuse, NY, USA, October 2013.

12

American Institute of Aeronautics and Astronautics 
${ }^{15}$ Henderson, J., Idris H., and Wing, D.J., "Preliminary Benefits Assessment of Traffic Aware Strategic Aircrew Requests (TASAR)", AIAA-2012-5684, AIAA 12 th Aircraft Technology, Integration, and Operations Conference (ATIO), Indianapolis, IN, USA, September 2012.

${ }^{16}$ Henderson, J., “Annualized TASAR Benefit Estimate for Virgin America Operations”, NASA/CR-2015-218786, NASA Langley Research Center, Hampton, VA, 2015.

${ }^{17}$ Henderson, J., “Annualized TASAR Benefit Estimate for Alaska Airlines Operations”, NASA/CR-2015-218787, NASA Langley Research Center, Hampton, VA, 2015.

${ }^{18}$ Karr, D. and Vivona, R., "Conflict Detection Using Variable 4D Uncertainty Bounds to Control Missed Alerts", AIAA-2006-6057, AIAA Guidance, Navigation and Control Conference, Keystone, CO, USA, 2006.

${ }^{19}$ Vivona, R., Karr, D., Roscoe, D., "Pattern-Based Genetic Algorithm for Airborne Conflict Resolution", AIAA2006-6060, AIAA Guidance, Navigation and Control Conference, Keystone, CO, USA, 2006.

${ }^{20}$ Karr, D., Vivona, R., Roscoe, D., DePascale, S., and Consiglio, M., "Experimental Performance of a Genetic Algorithm for Airborne Strategic Conflict Resolution”, AIAA Guidance, Navigation and Control Conference, Chicago, IL, USA, 2009.

${ }^{21}$ Karr, D.A., Vivona, R.A., and Wing, D.J., "Costs of Limiting Route Optimization to Published Waypoints in the Traffic Aware Planner”, AIAA Guidance, Navigation and Control Conference, Boston, MA, USA, August 2013.

${ }^{22}$ Vivona, R.A., Cate, K.T., and Green, S.M., “Abstraction Techniques for Capturing and Comparing Trajectory Predictor Capabilities and Requirements", Proceedings of the 2008 AIAA Conference on Guidance, Navigation, and Control, Honolulu, HI.

13

American Institute of Aeronautics and Astronautics 